\title{
Application of the Bees Algorithm for Constrained Mechanical Design Optimisation Problem
}

\author{
Shafie Kamaruddin and Mohd Arif Hafizi Abd Latif
}

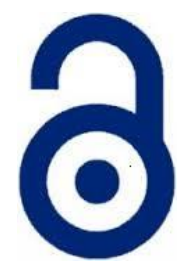

\author{
Received: 12 November 2018 \\ Accepted: 09 January 2019 \\ Published: 01 March 2019 \\ Publisher: Deer Hill Publications \\ (C) 2019 The Author(s) \\ Creative Commons: CC BY 4.0
}

\begin{abstract}
Optimisation is a technique or procedure to find the optimal or feasible solution whether it is to minimise or maximise by comparing other possible solutions until the best solution is found. Nowadays, many optimisation algorithms have been introduced due to the advancement of technology such as Teaching Learning Based Optimisation (TLBO), Ant Colony Optimisation (ACO), Particle Swarm Optimisation (PSO) and the Bees Algorithm. The Bees Algorithm is considered as one of the best optimisation algorithms because it has been successfully solved different type optimisation problem from in various field. It is inspired by the foraging behaviour of honeybees in nature. This study applies the Bees Algorithm to minimise the mass of disc clutch brake in its design. To find the optimal solution for the multiple disc clutch design, the Bees Algorithm is applied and found better result compared to other optimisation algorithms that already have been used.
\end{abstract}

Keywords. Bees Algorithm, Optimisation Algorithm, Multiple Disc Clutch Problem

\section{INTRODUCTION}

In this era, many problems need optimisation regardless to minimise or maximise. For examples, minimise (cost, weight, time) and maximise (profit, performance). Optimisation technique is a procedure to find the optimal solution whether it is minimisation or maximisation by comparing other possible solutions until the satisfactory solution is found. This technique finds the combination of parameters or variables to maximise or minimise objective functions subject to some constraints. One of the methods to solve the optimisation problem is using optimisation algorithms. There are many types of algorithms available today to find the optimal solution such as Ant-Colony algorithm, the Bees Algorithm, Teaching-Learning Based Optimisation and Particle Swarm Optimisation.

The Bees Algorithm is considered as among the best optimisation algorithms because it has been successfully solved different types of optimisation problems (Hussein et al., 2016). It is inspired by the foraging behaviour of honeybees in nature. Multiple Disc Clutch Brake problem is one of the common problems faced in real world problem and it has been applied by other algorithms. This problem requires finding the best combination of design variables (optimal design) at a minimum weight. Despite it has been applied by other algorithms, applying the Bees Algorithm on this problem would be interesting in term of exploring the capability of the Bees Algorithm in different problem. The significant of this research output would contribute in finding best design variables at minimum weight compared with other algorithms, which will improve the efficiency in product design management. For this reason, this problem was selected to test the performance of the Bees Algorithm. This study also provides other alternative algorithm in solving this problem. The main objective of this problem is to find the best combination of variables of Multiple Clutch Brake design with minimum mass and satisfy all the constraints.

\section{THE BEES ALGORITHM}

\subsection{Bees in Nature}

A colony of bees always has some of its population to be used as scout bees to scrutinize the surrounding of their hive for potential patches of flower. The process of foraging starts when the scout bees are sent to the space and move randomly from one patch to another patch. Then, the scout bees return to the hive and inform other bees in

\section{S. Kamaruddin $\otimes$ and M. A. H. A. Latif}

Department of Manufacturing and Materials Engineering

International Islamic University Malaysia

PO Box 10, 50728 Kuala Lumpur, Malaysia

E-mail: shafie@iium.edu.my

Reference: Kamararuddin and Latif (2019). Application of the Bees Algorithm for Constrained Mechanical Design Optimisation Problem. International Journal of Engineering Materials and Manufacture, 4(1), 27-32. 
the hive about the quality and location of a food source by performing a dance called "waggle dance". It will recruit other bees to exploit high potential location while other scout bees will continue to find new patches (Pham et al., 2009).

In this foraging behaviour, the bees need to divide their workforce to each task with proper number of bees. They also must flexible enough to adapt to any environment changes. There are many mechanisms on how a colony of bees regulates the division of labour between the scouts and the recruits (Beekman et al., 2007). Based on these behaviours, several bees inspired algorithms have been established such as Artificial Bee Colony (ABC) algorithm, Bee Colony Optimisation algorithm (BCO) and the Bees Algorithm (Karaboga \& Basturk, 2007; Nikolić \& Teodorović, 2013; Pham \& Castellani, 2015). Although, all these algorithms are similar in term of inspiration from bees foraging behaviour, but each algorithm has its own work mechanism. This study focuses on one of the algorithms, which is the Bees Algorithm.

\subsection{Description of the Bees Algorithm}

The Bees Algorithm uses the same concept as the food foraging behaviour of honey bees in the nature. It was introduced by a group of researchers from Cardiff University (Pham et al., 2006). It works by sending the scouts bees to explore for potential solutions. Then, the potential sites found are exploited and better solution will attract more recruits. This process continues iteratively until the best solution has been found. The flow chart of the Bees Algorithm is shown in Figure 1 (Pham \& Castellani, 2009).

The first step of the Bees Algorithm is setting the parameters which are number of scout bees ( $n$ ), number of best sites selected out of $n$ sites $(m)$, number of elite sites selected out of best sites $(e)$, number of bees recruited for elite sites (nep), number of bees recruited for the other selected sites ( $n s p)$, patches size ( $n g h)$ and number of unselected scout bees $(n-m)$. The next step of the Bees Algorithm is sending $n$ scout bees randomly (random initialisation) across the search space. After that, each position visited by the scout bee is evaluated via fitness function and ranked according to it fitness value. Once the positions visited by the scout bees have been ranked, local search is performed by sending recruit bees ( $n e p$ and $n s p$ ) to selected elite sites and best sites. Elite sites attract more recruit bees compared to best sites. These recruit bees are placed randomly across patch size of the selected sites. If the recruit bees found better fitness value compared to the followed scout bee of the selected site, it will replace the scout bee as the new scout bee. If the recruit bees failed to find better fitness value, the scout bee remains as scout bee for that patch. As for the remaining unselected scout bees, they are sent randomly across the space to find new patches (global search). At the end of each iteration, a new population is formed consists of best recruit bees from each patch and unselected scout bees. The stopping criterion can be set either based on predefined threshold or predefined number of iteration (Pham \& Castellani, 2009).

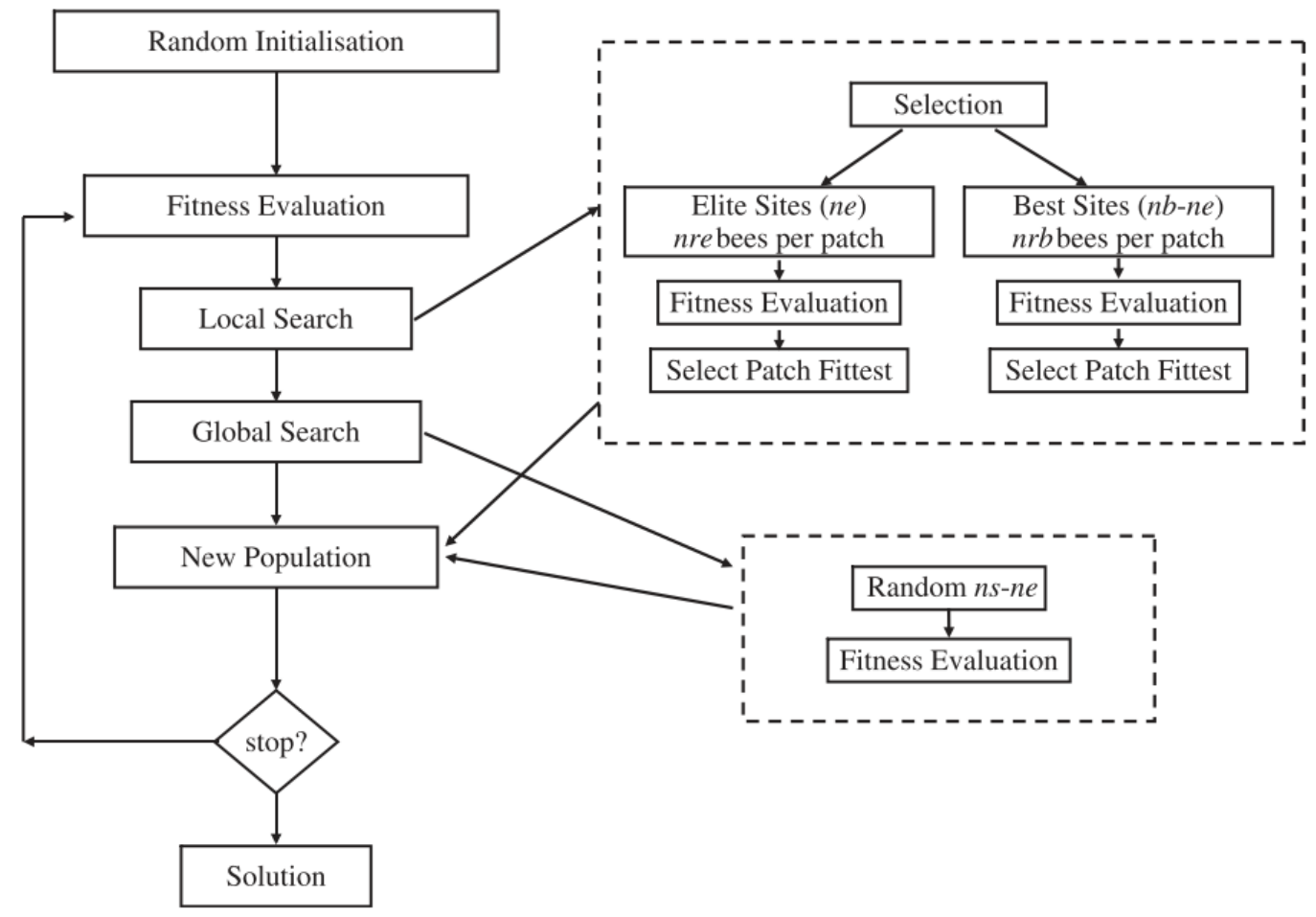

Figure 1: Flowchart of the Bees Algorithm 


\subsection{Multiple Disc Clutch Problem}

The objective is to minimise the mass of the multiple disc clutch brake design, which consists of five discrete variables; inner radius, $r$, outer radius, $r$, thickness of the discs, $t$, actuating forces, $F$, and number of friction surfaces, $Z$ (Rao et al., 2011). Figure 2 shows the schematic of Multiple Disc Clutch used for this application. The objectives function and constraints for this problem are shown in Appendix.

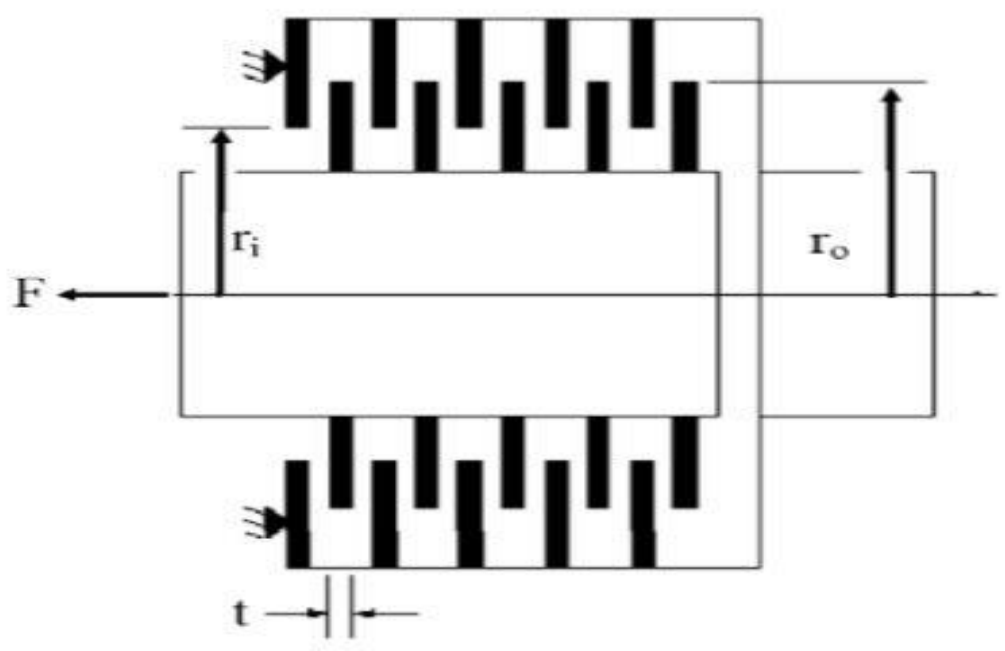

Figure 2: Multiple Disc Clutch Schematic

\section{METHODOLOGY}

This study started with background study or reviewing the articles, journals and books related to the problem. The articles are about the optimisation algorithm, specifically the Bees Algorithm and the mechanical design problem, which is multiple disc clutch.

Then, writing the code in open source software known as $R$-Software and run the code. Most of optimisation algorithms require fine-tuning of parameters to find the best solution. Thus, several set of parameters were selected for this experiment. For each set of parameters, the algorithm was run 100 times. The result of best fitness value, worst fitness value, mean, standard deviation and successful rate found over 100 runs of each set of parameters were recorded. The criteria in determining the best result is based on the mean of fitness values found over 100 runs. The set of parameters that generated the best result are shown in Table 1.

The stopping criterion for this experiment was set based on number of function evaluations, which are 1000 function evaluations. This means the algorithm was stopped once it reached maximum number of evaluations for each run. Next, results of this experiment were compared with other optimisation algorithms available in the literature. The best result found was compared with other optimisation algorithms available in the literature. Most of results in the literature do not provide the standard deviation value of their results. Thus, the comparison is in term of mean value only. The value of variables and constraints are also shown and compared between those different optimisation algorithms.

Table 1: Set of Parameters of the Bees Algorithm

\begin{tabular}{cc}
\hline Parameters & Value \\
\hline$n$ & 14 \\
$e$ & 2 \\
$n e p$ & 11 \\
$n g h$ & 3 \\
$m$ & 4 \\
$n s p$ & 4
\end{tabular}




\section{RESULTS AND DISCUSSIONS}

4.1 Results

The multiple disc clutch brake design problem was also solved by NSCA-II, TLBO, ABC, and APSO. Table 2 shows the statistical comparisons of other optimisation algorithm results with the Bees Algorithm. The best value of the mass of the multiple disc clutch brake found by the Bees Algorithm is $0.313657 \mathrm{~kg}$. This value is similar with TLBO and $A B C$, but better compared to the results of NSGA-II and APSO.

Table 3 shows the combination of variables found by the Bees Algorithm corresponding to the best fitness value over 100 runs. In most cases, the combination of variables is similar with other algorithms as the best fitness value is similar with TLBO and ABC. Despite statistical result, comparison shows that TLBO achieved better successful rate (SR) compared to the Bees Algorithm, but the Bees Algorithm showed less variability of the result with standard deviation only 0.008631. In terms of other statistical results such as worst and mean fitness values found, the Bees Algorithm also shows better results compared with other optimisation algorithms. Figure 3 and Figure 4 show the comparison of convergence rate for the Bees Algorithm and $A B C$ algorithm respectively. From Figure 4, it is observed that the convergence rate for the TLBO method is faster than $A B C$ in the early stage of the search. Then, both found almost similar fitness value until at the end of the search. This could have contributed to better successful rate of TLBO compared to ABC algorithm and the Bees Algorithm.

Table 2: Result Comparison with other Optimisation Algorithms

\begin{tabular}{llllll}
\hline & Best & Worst & Mean & SR & SD \\
\hline TLBO (Rao et al., 2011) & 0.313657 & 0.392071 & 0.327166 & 0.67 & N/A \\
ABC (Rao et al., 2011) & 0.313657 & 0.352864 & 0.324751 & 0.54 & N/A \\
NSCA-II (Debb \& Srivinasan, 2006) & 0.4704 & N/A & N/A & N/A & N/A \\
APSO (Ben Guedria, 2016) & 0.337181 & 0.716313 & 0.506829 & N/A & 0.09767 \\
Bees Algorithm & 0.313657 & 0.345022 & 0.320761 & 0.41 & 0.008631 \\
\hline
\end{tabular}

Table 3: Values of Objectives Functions, Design Variables and Constraints

\begin{tabular}{cccccc}
\hline Variables & $\begin{array}{c}\text { TLBO } \\
\text { (Rao et al., 2011) }\end{array}$ & $\begin{array}{c}\text { NSCA-II } \\
\text { (Debb \& } \\
\text { Srivinasan, } \\
2006)\end{array}$ & $\begin{array}{c}\text { ABC } \\
\text { (Rao et al., 2011) }\end{array}$ & $\begin{array}{c}\text { APSO } \\
\text { (Ben Cuedria, } \\
2016)\end{array}$ & $\begin{array}{c}\text { Bees } \\
\text { Algorithm }\end{array}$ \\
\hline$x 1$ & 70 & 70 & N/A & 76 & 70 \\
$x 3$ & 90 & 90 & N/A & 96 & 90 \\
$x 4$ & 1 & 1.5 & N/A & 1 & 1 \\
$x 5$ & 810 & 1000 & N/A & 840 & 990 \\
$f(x)$ & 0.313657 & 0.4704 & N/A & 3 & 3 \\
$g 1$ & 0 & 0 & 0.313657 & 0.337181 & 0.313657 \\
$g 2$ & 24 & 22 & N/A & 0 & 0 \\
$g 3$ & 0.919428 & 0.9005 & N/A & 24 & 20 \\
$g 4$ & 9830.371 & 9.7906 & N/A & 0.922273 & 999.902 \\
$g 5$ & 7894.697 & 7.894 & N/A & 9.824211 & 9999793 \\
$g 6$ & 0.702013 & 3.3527 & N/A & 7.738378 & 7894.697 \\
$g 7$ & 37706.25 & 60.625 & N/A & 1.396611 & 3.23794 \\
$g 8$ & 14.29799 & 11.6473 & N/A & 48.84837 & 59418.75 \\
\hline
\end{tabular}




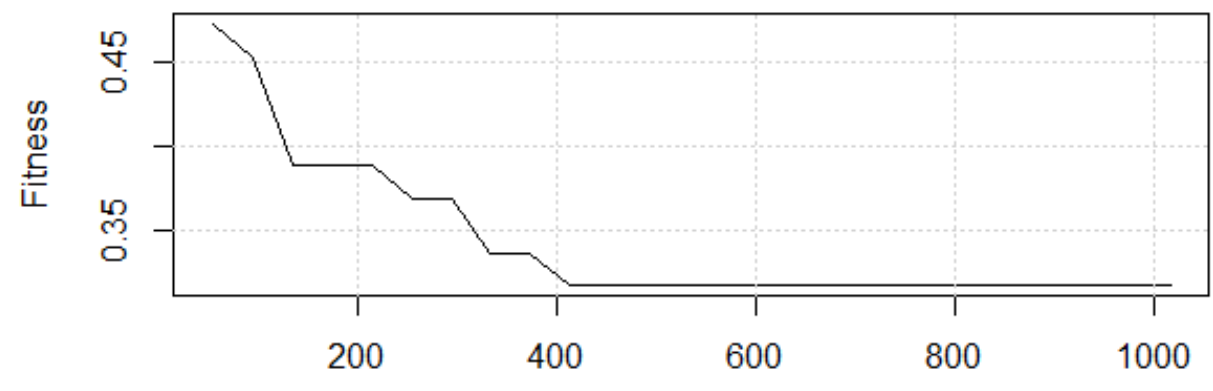

Numbers of function evaluation

Figure 3: Convergence plots for the Multiple Disc Clutch Brake using Bees Algorithm

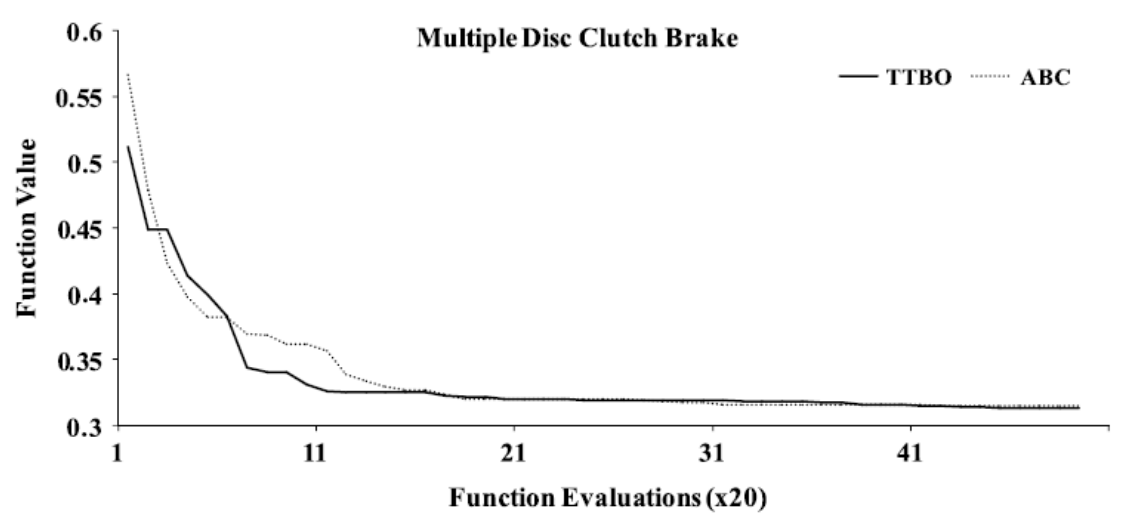

Figure 4: Convergence plots for the Multiple Disc Clutch Brake using the TLBO and ABC algorithms (Rao et al., 2011).

\subsection{Discussions}

The results of the Bees Algorithm were compared with the results of other algorithms to compare it performance. Some of these optimisation algorithms also share almost similar work mechanism with Bees Algorithm. The $A B C$, APSO and the Bees Algorithm are in the same category of Swarm-based optimisation. The Bees Algorithms and ABC use the concept of exploration and exploitation, while APSO focuses more on exploration. Meanwhile, NSCA-II and TLBO have their own search mechanisms. TLBO uses the concept of learning process in a class with certain number of student (population) and subject (variable) and NCSA is a type of evolutionary algorithm.

As shown it Table II, it is observed that three out of five (ABC, TLBO and The Bees Algorithm) optimisation algorithms able to find similar best solution, which is $0.313657 \mathrm{~kg}$. The remaining two algorithms (NCSA-II and APSO) found worse solutions where, APSO obtained $0.337181 \mathrm{~kg}$ and NSGA-II obtained $0.4704 \mathrm{~kg}$. In term of mean's value and standard deviation's value, the Bees Algorithm achieved better value, which is $0.320761 \mathrm{~kg}$ and 0.008631 respectively. The successfulness of the Bees Algorithm and $A B C$ algorithm in finding the best solution could due their similarity of work.

\section{CONCLUSIONS}

In this work, a swarm based optimisation algorithm named the Bees Algorithm is applied to constrained mechanical design problem (Multiple Clutch Brake problem) and its performance is compared with other algorithms. The results obtained show that the Bees Algorithm found similar best fitness value compared with other algorithms at similar computational effort (number of function evaluation). Despite the best fitness, value found is similar with other optimisation algorithms such as TLBO algorithm and ABC algorithm, the Bees Algorithm has fewer variations with better mean and standard deviation. In conclusion, this research showed:

1. The successfulness of applying the Bees Algorithm to find the best solutions for mechanical design optimisation problem.

2. Despite the best fitness value is similar with other algorithms, the Bees Algorithm found better results in term of mean and standard deviation which are $0.320761 \mathrm{~kg}$ and 0.008631 respectively.

3. The capability of applying the Bees Algorithm to more complex optimisation problem. 


\section{ACKNOWLEDGEMENT}

The authors are grateful to the Department of Manufacturing and Materials Engineering, International Islamic University Malaysia for giving the opportunity to complete this study.

\section{APPENDIX: OBJECTIVE FUNCTIONS AND CONSTRAINTS}

The objective is to minimize the mass of the multiple disc clutch brake using five discrete variables: inner radius $\left(r_{\mathrm{i}}=60,61,62, \ldots, 80\right)$, outer radius $\left(r_{\mathrm{o}}=90,91,92, \ldots, 110\right)$, thickness of discs $(t=1,1.5,2,2.5,3)$, actuating force $(F=600,610,620, \ldots, 1000)$ and number of friction surfaces $(Z=2,3,4,5,6,7,8,9)$.

Minimise: $f(x)=\pi\left(r_{0}^{2}-r_{l}^{2}\right) t(Z+1) \rho$

Subject to: $g_{1}(x)=r_{0}-r_{1}-\Delta r \geq 0$,

$$
\begin{aligned}
& g_{2}(x)=I_{\max }-(Z+1)(t+\delta) \geq 0, \\
& g_{3}(x)=p_{\max }-p_{r z} \geq 0, \\
& g_{4}(x)=p_{\max } v_{s r m a x}-p_{r z} v_{s r} \geq 0, \\
& g_{5}(x)=v_{s r \max }-v_{s r} \geq 0, \\
& g_{6}(x)=T_{\max }-T \geq 0, \\
& g_{7}(x)=M_{h}-s M_{s} \geq 0, \\
& g_{8}(x)=T \geq 0,
\end{aligned}
$$

where $M_{h}=\frac{2}{3} \mu F Z \frac{r_{o}^{3}-r_{i}^{3}}{r_{o}^{2}-r_{i}^{2}}, p_{r z}=\frac{F}{\pi\left(r_{o}^{2}-r_{i}^{2}\right)}, V_{s r}=\frac{2 \pi n\left(r_{o}^{3}-r_{i}^{3}\right)}{90\left(r_{o}^{2}-r_{i}^{2}\right)}, \quad T=\frac{I_{z} \pi n}{30\left(M_{h}+M_{f}\right)}$,

$\Delta r=20 \mathrm{~mm}, t_{\max }=3 \mathrm{~mm}, t_{\min }=1.5 \mathrm{~mm}, I_{\max }=30 \mathrm{~mm}, Z_{\max }=10, v_{s r \max }=10 \mathrm{~m} / \mathrm{s}, \mu=0.5, \mathrm{~s}=1.5, M_{s}=40$ $\mathrm{N} \mathrm{m}, M_{f}=3 \mathrm{~N} \mathrm{~m}, n=250 \mathrm{rpm}, p_{\max }=1 \mathrm{MPa}, l_{z}=55 \mathrm{~kg} \mathrm{~mm}^{2}, T_{\max }=15 \mathrm{~s}, F_{\max }=1000 \mathrm{~N}, r_{\text {inin }}=55 \mathrm{~mm}$, $r_{\text {omax }}=110 \mathrm{~mm}$

\section{REFERENCES}

1. Beekman, M., Gilchrist, A. L., Duncan, M., \& Sumpter, D. J. T. (2007). What makes a honeybee scout? Behavioral Ecology and Sociobiology, 61(7), 985-995.

2. Ben Guedria, N. (2016). Improved accelerated PSO algorithm for mechanical engineering optimisation problems. Applied Soft Computing Journal, 40, 455-467.

3. Deb, K., \& Srinivasan, A. (2006). Innovization: Innovating design principles through optimisation. Proceedings of the 8th Annual Conference on, 1629-1636.

4. Hussein, W. A., Sahran, S., \& Sheikh Abdullah, S. N. H. (2016). The variants of the Bees Algorithm (BA): a survey. Artificial Intelligence Review, 1-55.

5. Karaboga, D., \& Basturk, B. (2007). A powerful and efficient algorithm for numerical function optimization: Artificial bee colony $(A B C)$ algorithm. Journal of Global Optimization, 39, 459-471.

6. Nikolić, M., \& Teodorović, D. (2013). Empirical study of the Bee Colony Optimization (BCO) algorithm. Expert Systems with Applications, 40(11), 4609-4620.

7. Pham, D. T., \& Castellani, M. (2009). The Bees Algorithm : Modelling foraging behaviour to solve continuous optimisation problems, Proceedings of the Institution of Mechanical Engineers, Part C: Journal of Mechanical Engineering Science, 223(12), 2919-2938.

8. Pham, D. T., \& Castellani, M. (2015). A comparative study of the Bees Algorithm as a tool for function optimisation. Cogent Engineering, 2(1), 1091540.

9. Pham, D. T., Ghanbarzadeh, A., Koc, E., Otri, S., Rahim, S., \& Zaidi, M. (2006). The Bees Algorithm-A Novel Tool for Complex Optimisation. Proceedings of the 2nd International Virtual Conference on Intelligent Production Machines and Systems, 454-459.

10. Pham, D. T., Ghanbarzadeh, A., Otri, S., \& Koç, E. (2009). Optimal design of mechanical components using the Bees Algorithm. Proceedings of the Institution of Mechanical Engineers, Part C: Journal of Mechanical Engineering Science, 223(5), 1051-1056.

11. Rao, R. V., Savsani, V. J., \& Vakharia, D. P. (2011). Teaching-learning-based optimisation: A novel method for constrained mechanical design optimisation problems. Computer-Aided Design, 43(3), 303-315. 